\title{
Comparison of cavitation bubbles evolution in viscous media
}

\author{
Darina Jasikova ${ }^{*}$, Petr Schovanec, Michal Kotek, Vaclav Kopecky \\ Department of Physical Measurement, Technical University of Liberec, The Institute for Nanomaterials, Advanced Technology and \\ Innovation, Studentska 1402/2 Liberec 1461 17, Czech Republic
}

\begin{abstract}
There have been tried many types of liquids with different ranges of viscosity values that have been tested to form a single cavitation bubble. The purpose of these experiments was to observe the behaviour of cavitation bubbles in media with different ranges of absorbance. The most of the method was based on spark to induced superheat limit of liquid. Here we used arrangement of the laser-induced breakdown (LIB) method. There were described the set cavitation setting that affects the size bubble in media with different absorbance. We visualized the cavitation bubble with a $60 \mathrm{kHz}$ high speed camera. We used here shadowgraphy setup for the bubble visualization. There were observed time development and bubble extinction in various media, where the size of the bubble in the silicone oil was extremely small, due to the absorbance size of silicon oil.
\end{abstract}

\section{Introduction}

The cavitation phenomena is mainly known for its undesirable effects in turbo machinery, however there is a great potential for its utilization in the industry, medicine, biology, pharmacy, or tissue engineering.

The research in the field of cavitation was previously focused mainly on the investigation of the bubble behavior in the vicinity of rigid or flexible boundaries; [1-4] however the current investigations require including the description of response of the impacted material itself. [1-2] The definition of the material response can help in development of new, more resistant structures or layers which better withstand the action of cavitation bubbles and prolong the service life of products. The key in the understanding of the cavitation interaction with various materials is the investigation of the impact of individual bubbles and its structures [1].

Cavitation can be defined as a collection of effects connected to the origin, activities, and collapse of macroscopic bubbles in liquid. Cavitation bubbles are usually not separated in real applications. The bubbles create structures, which acts collectively, however essential elements of these structures are individual bubbles [2].

Although the current state of technique is at very high level, we are still not able to produce one controllable individual bubble by pressure decrease in the liquid volume, following the cavitation definition. All other methods as the spark or laser generated bubbles are closer to the boiling as these are based on the evaporation of small volume of liquid. The bubbles generated by the ultrasonic field satisfy the cavitation definition, however to produce one single bubble is almost impossible. Anyway, we dealing with great technique LIB, there still exists a lack of information in experimental part of cavitation bubble investigation [1].

The one of the method for bubble generation is spark in liquid, or a heated top of a wire. Once the bubble is stable, and of certain volume, it is either over heated, or expose to force impact. The convenient method for generating single cavitation bubble that can be very precisely geometrically placed in the volume of the liquid, and close to the sample, is Laser Induced Breakdown (LIB). [5-9] The LIB method enables to generate cavitation bubble using ultrashort pulses of milijoules energies. This method is thermal breakdown based on natural plasma generation. This plasma is in form of optical breakdown, if the pulse exposure is from microseconds to femtoseconds' time. There occurs direct, multiphoton, and cascade ionization during the LIB. The significant role plays impurities of the medium, spot size, light wavelength, and pulse width during the breakdown. The whole mechanism of the ionization is very well explained by Kennedy [6].

The research of bubble behavior focuses primarily on the movement of bubbles in viscous liquids. The motion of bubbles in fluids is of great importance in various gas-liquid reactors and processes, as well as numerous natural phenomena. As a result, extensive studies have been conducted in the past (see reviews by Clift et al. (1978), Magnaudet and Eames (2000), and Kulkarni and Joshi (2005)), although various aspects still remain indeterminate, particularly in relation to the dynamic behaviour of the bubbles in liquids [10].

Bubble generated in a higher viscosity liquid is being formation in two differs stages. For the evolution of the volume of the bubble in the breakup stage is always greater than that in the expansion stage, due to the different role of the liquid phase in the evolution of the bubble for the two stages [11]. 
Furthermore, the effect of absorbance and absorption of light on bubble generation in the liquid is important, where absorption of light in the sample is described in the Lambert-Beer Law. The measured values are expressed as absorbance or transmittance. Absorption is usually measure in a linear arrangement. Absorption spectrometers: single-beam or two-beam. The intensity of the beam passing through the sample also weakens scattering. The measurement gives reasonable results for the absorbance in the interval $(0.005-1)$ [12].

Absorbance takes values from 0 to infinity. If the absorbance has a zero value, the substance does not absorb any radiation and the permeability is equal to one. If the absorbance is infinite, the substance under investigation absorbs all the radiation and the throughput is zero. It should also be noted that less than $10 \%$ of the light passes through the absorbance of more than 1 , and a small error in passing radiation can cause a large error in the determination of the absorbance. Most determinations are therefore performed so that the maximum absorbance value lies around the value of 1 [13].

\section{Experimental}

The bubbles can be generated by several physically different mechanisms. The most obvious in nature is the hydrodynamics cavitation, where bubbles are produced due to local pressure decrease caused by the flow acceleration in vicinity of obstacles. Acoustic cavitation is produced by imposing an intensive acoustic field into the bulk of liquid [5].

Here, we used laser induced breakdown for cavitation bubble generating in liquid. Experiments run in optical glass cuvette.

\subsection{Laser Induced Breakdown}

Optical breakdown in liquid is usually produced by focusing of the laser light trough suitably designed optics. Laser induced breakdown in aqueous media and its collateral effects are described in detail by Kennedy in [6]. The energy distribution during the growth and collapse of laser induced bubble was described e.g. by Vogel in [14] and [15]. Authors investigated the influence of laser pulse duration and input laser energy on the bubble dynamics and shock waves emission.

Here we chosen green light as exciting light pulse. The absorption coefficient of the $532 \mathrm{~nm}$ laser light is only $0.02 \mathrm{~m}^{-1}$ in distillated pure water. This wave length requires higher energy input to the system to induce cavitation process. For the single bubble generation we used here the setup for the LIB. The $10 \mathrm{~ns}$ short laser pulse was generated using Q-switched Nd: YAG New Wave Gemini pulse laser. This laser worked with one cavity for single shot generation on the wavelength 532 nm. The Q-switch signal synchronized the high speed camera running in triggering mode.

LIB was set as an optical direct way. The outlet diameter of laser beam was $5 \mathrm{~mm}$ with Gaussian characteristics of the intensity. This setup was followed with concave lens $f_{s}=200 \mathrm{~mm}$, and convex lens $f_{s}=25$ $\mathrm{mm}$ of 1 inch diameter. The focused laser beam created the laser point - probe (diameter $<0.1 \mathrm{~mm}$ ). Due the losses in the optical path on each of optical elements, comparable to this, we had to increase the energy level that enters the whole system. The set output energy of the laser is taken in account in the relation to the bubble diameter. The energies that are required for the bubble generation are seen in Fig. 1.

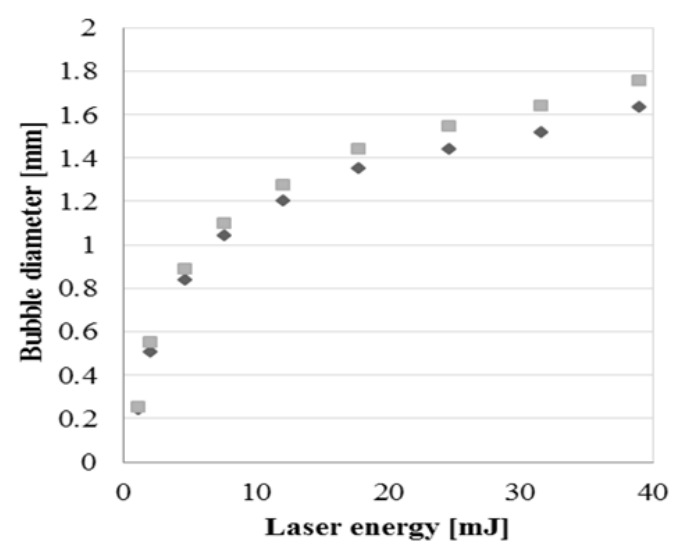

Fig. 1. The graph of maximal bubble diameter on laser energy.

For the relation between input laser energy and the bubble size that was generated with our optical setup, we performed the set of calibration and size measurement. The energy of the laser beam was adjusted and measured with Ophir pyroelectric energy sensor. We measured the laser energy in three positions. There is seen the characteristic of laser light energy calibrated to the position of attenuator. NewWave Gemini laser enables setting of light intensity adjusting flash lamp power and fine adjustment using attenuator. The attenuator enables controllable backward loop, as an actual value is seen on the display. We set the laser on full flash lamp power and adjust the output energy using attenuator. It is seen the energy disorder in Fig. 2, when the attenuator is 0 , the output laser energy is higher than for attenuator 50 . We neglect this effect as we are working on the attenuator position starting on 120 to generate cavitation bubble, anyway. We suppose this is laser attenuator characteristic.

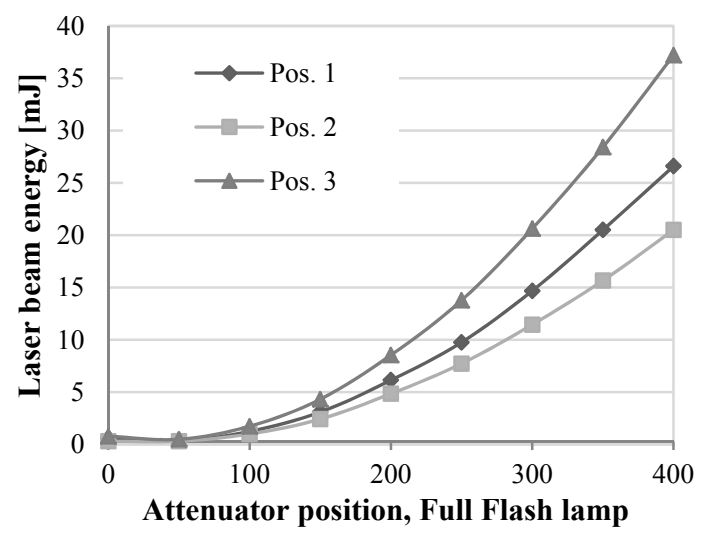

Fig. 2. The calibration curve of output energy on laser attenuator; Position 1 was at the outcome of the laser head, 
position 2 was after the collimating lens, and position 3 was after the focusing mirror

According to Kennedy [16], plasma temperature shows asymptotic dependence on laser pulse energy as well. With higher laser energy we recognized negative influence of impurities and presence of segmentations on the bubble surface.

\subsection{Optical setup}

Here we used two kind of setup for LIB: the direct way with $50 \mathrm{~mm}$ Plano-Convex Lens and $30 \mathrm{~mm}$ PlanoConvex Lens. The direct optical setup enables easy handling and replacement of single optical component. Here we work with the lowest possible number of optical components, so we expecting lowest light intensity lost in system. The setup and used optical components is seen in Fig. 3.

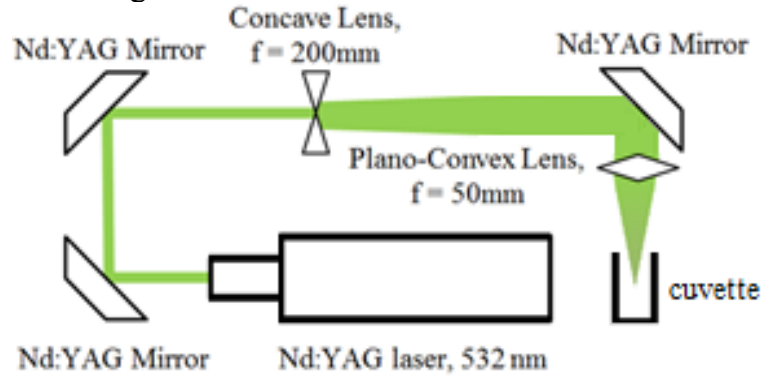

Fig. 3. The optical setup of LIB technique for generating cavitation bubble.

\subsection{Visualization setup}

We used here shadowgraphy setup for the bubble visualization. This setup consists of continuous LED matrix daylight lamp Veritas, MiniConstellation 120 $5000 \mathrm{~K}$ of illuminance $92 \mathrm{klux}$ in $0.5 \mathrm{~m}$, set with optical diffuse filter. Opposite to the light source was placed high speed CMOS camera SpeedSense. This camera is working on frequency $60 \mathrm{kHz}$ with resolution of $(128 \mathrm{x}$ 128) px or lower frequency with higher resolution up to $(1280 \times 800) \mathrm{px}$, and the dynamic range 12 bit.

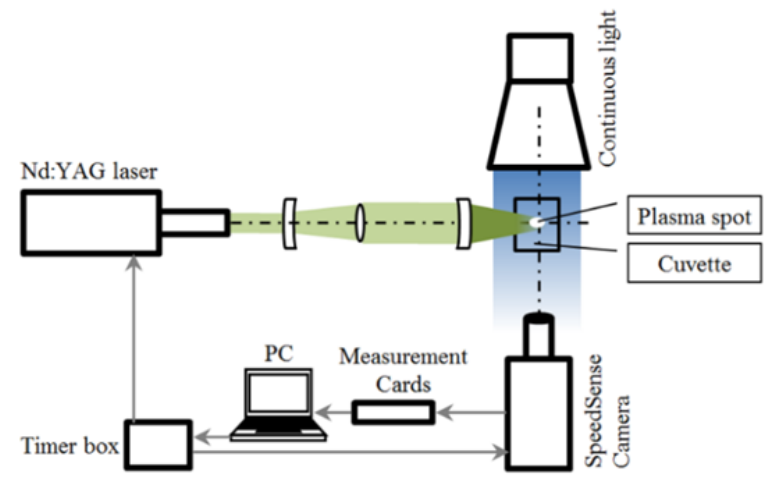

Fig. 4. The experimental setup of the laser, and the visualization system.

The camera exposure time was $1 \mu$ s. The sub-pixel resolution was $20 \mu \mathrm{m}$. The camera was mounted with optical lens system INFINIPROBETM TS-160 universal macro/micro imaging system that enables $4 x$, and $16 x$ magnification. The camera was mounted with edge pass and long pass filter cut-on wavelength $550 \mathrm{~nm}$ low pass optical filter to reduce the backward laser flashes to the camera and also to eliminate the flash generated while plasmatic breakdown.

We also used a light reflecting setup for visualization of the outer bubble structures. We placed the light under $45^{\circ}$ from the camera direction.

\subsection{Absorbance}

The Lambert-Beer law expresses the relationship between the absorbance and the concentration of the investigated substance.

$$
A=\varepsilon . c . l
$$

where $\mathrm{A}$ is the absorbance, c molar concentration of the test sample $(\mathrm{mol} / \mathrm{l}), 1$ cell thickness $(\mathrm{cm})$ and $\varepsilon$ molar absorption coefficient $\left(1 . \mathrm{mol}^{-1} \cdot \mathrm{cm}^{-1}\right)$. The molecular absorption coefficient is a constant for a given substance at a given wavelength [13].

\begin{tabular}{|l|c|c|c|}
\hline Liquid & $\begin{array}{c}\text { Ethyl } \\
\text { Alcohol }\end{array}$ & $\begin{array}{c}\text { Ethylene } \\
\text { Glycol }\end{array}$ & Glycerine \\
\hline $\begin{array}{l}\text { Surface tension } \\
{[\mathrm{mN} / \mathrm{m}], \text { temp. }}\end{array}$ & $\begin{array}{c}22,39 \\
{\left[20^{\circ} \mathrm{C}\right]}\end{array}$ & $\begin{array}{c}47,30 \\
{\left[25^{\circ} \mathrm{C}\right]}\end{array}$ & $\begin{array}{c}63,4 \\
{\left[20^{\circ} \mathrm{C}\right]}\end{array}$ \\
\hline $\begin{array}{l}\text { Kinematic } \\
\text { viscosity }\left[\mathrm{mm}^{2} / \mathrm{s}\right]\end{array}$ & $\begin{array}{c}1,52 \\
{\left[20^{\circ} \mathrm{C}\right]}\end{array}$ & $\begin{array}{c}17,80 \\
{\left[21,1^{\circ} \mathrm{C}\right]}\end{array}$ & $\begin{array}{c}648 \\
{\left[20,3^{\circ} \mathrm{C}\right]}\end{array}$ \\
\hline $\begin{array}{l}789 \\
\text { Density }\left[\mathrm{kg} / \mathrm{m}^{3}\right]\end{array}$ & $\begin{array}{c}1097 \\
{\left[25^{\circ} \mathrm{C}\right]}\end{array}$ & $\begin{array}{c}1259 \\
{\left[25^{\circ} \mathrm{C}\right]}\end{array}$ \\
\hline $\begin{array}{l}\text { Absorption coeff. } \\
{\left[\mathrm{cm}^{-1}\right],}\end{array}$ & 0,0182 \\
wavelength & 0,0113 & 0,9837 \\
{$[535 \mathrm{~nm}]$} & {$[535 \mathrm{~nm}]$} & {$[1200 \mathrm{~nm}]$} \\
\hline
\end{tabular}

\begin{tabular}{|l|c|c|c|}
\hline Liquid & $\begin{array}{c}\text { Water } \\
\text { distilled }\end{array}$ & $\begin{array}{c}\text { Water } \\
\text { fresh }\end{array}$ & $\begin{array}{c}\text { Silicone } \\
\text { oil }\end{array}$ \\
\hline $\begin{array}{l}\text { Surface tension } \\
{[\mathrm{mN} / \mathrm{m}], \text { temp. }}\end{array}$ & $\begin{array}{c}72,80 \\
{\left[20^{\circ} \mathrm{C}\right]}\end{array}$ & $\begin{array}{c}72,86 \\
{\left[20^{\circ} \mathrm{C}\right]}\end{array}$ & $\begin{array}{c}20,10 \\
{\left[25^{\circ} \mathrm{C}\right]}\end{array}$ \\
\hline $\begin{array}{l}\text { Kinematic } \\
\text { viscosity }\left[\mathrm{mm}^{2} / \mathrm{s}\right]\end{array}$ & $\begin{array}{c}1,0038 \\
{\left[20^{\circ} \mathrm{C}\right]}\end{array}$ & $\begin{array}{c}1,13 \\
{\left[15,6^{\circ} \mathrm{C}\right]}\end{array}$ & $\begin{array}{c}16,50 \\
{\left[40^{\circ} \mathrm{C}\right]}\end{array}$ \\
\hline Density $\left[\mathrm{kg} / \mathrm{m}^{3}\right]$ & $\begin{array}{c}0,9980 \\
{\left[20^{\circ} \mathrm{C}\right]}\end{array}$ & $\begin{array}{c}0,9982 \\
{\left[20^{\circ} \mathrm{C}\right]}\end{array}$ & $\begin{array}{c}980 \\
{\left[25^{\circ} \mathrm{C}\right]}\end{array}$ \\
\hline $\begin{array}{l}\text { Absorption coeff. } \\
{\left[\mathrm{cm}^{-1}\right],}\end{array}$ & $\begin{array}{c}0,0002 \\
\text { wavelength }\end{array}$ & $\begin{array}{c}0,0006 \\
{[535 \mathrm{~nm}]}\end{array}$ & $\begin{array}{c}2211 \\
{[535 \mathrm{~nm}]}\end{array}$ \\
\hline
\end{tabular}

\section{Results}

There is generated the plasma in the spot due the concentration of the laser energy. The plasma is visible due the emission. The temperature in liquid increase to $10^{3} \mathrm{~K}$, and pressure to $10^{3}$ bar in the spot volume. This leads to plasma expansion at supersonic velocities, producing an acoustic shock wave following with cavitation bubble effect. It is important to mark for the following figures $5-7$ that the laser light comes from the up side on the pictures.

There have been discovered that at the same energy of the laser the bubble has a different lifetime depending 
on the type of liquid. Here we expected the bubble generated in liquid with the lower absorption coefficient to have the longest lifetime.

\begin{tabular}{|c|c|c|}
\hline Time & Glycerine & Ethyl Alcohol \\
\hline & $1 \mathrm{~mm}$ & $1 \mathrm{~mm}$ \\
\hline $\mathrm{t}_{0}=0 \mu \mathrm{s}$ & & i \\
\hline
\end{tabular}
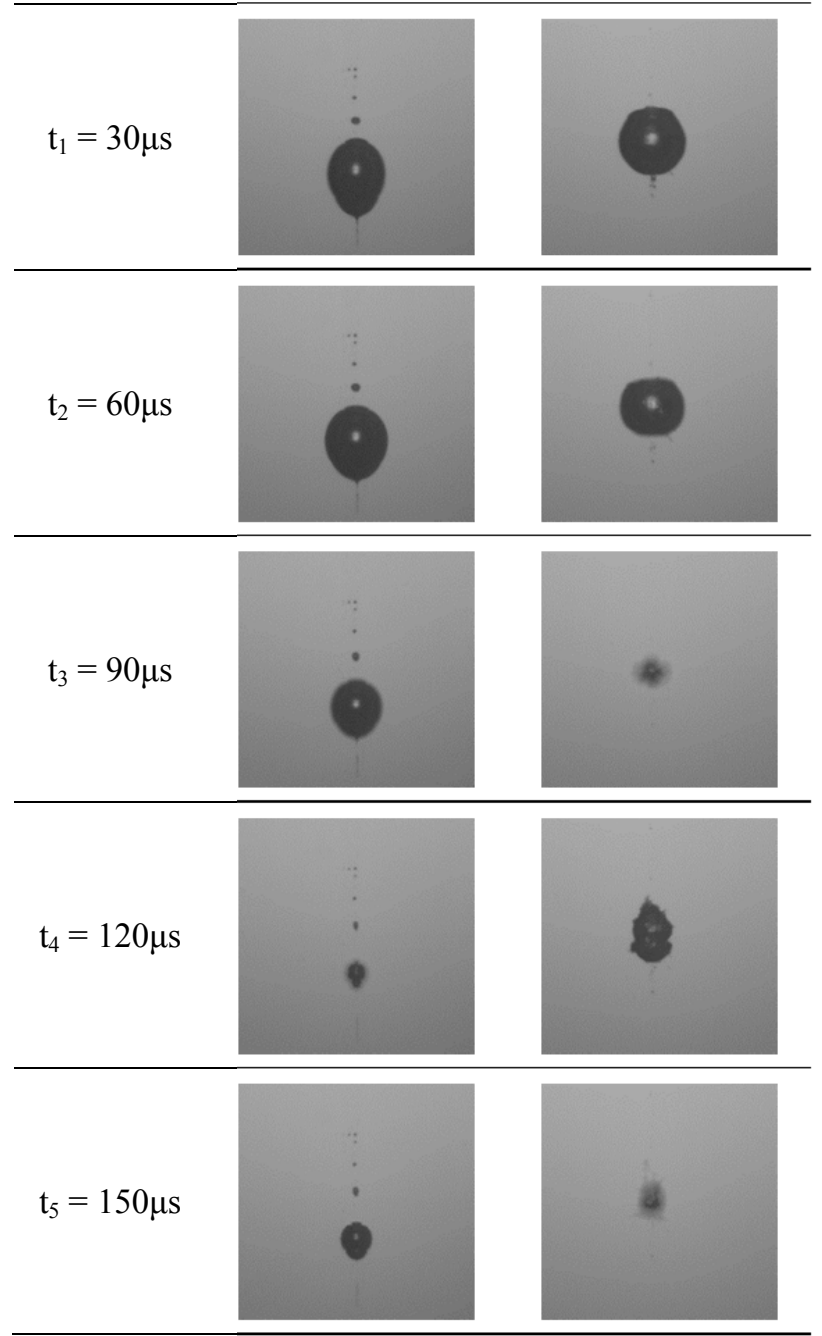

Fig. 5. Comparison of LIB generated bubble in Glycerine and Ethyl Alcohol for laser input energy $5,6 \mathrm{~mJ}$.

Figure 5 shows the development of the bubble in Glycerine and Ethyl Alcohol, where the size of the bubble is identical but its lifetime is different. Figure 6 shows the development of the bubble in Ethylene Glycol and water, where the size of the bubble and its lifetime is different.

It can be seen that the input laser beam differs in Figures 5 and 6 at time $t_{0}$ at the same laser energy. Figure 7 shows the bubble generated in Ethylene Glycol at double the energy of the laser and an interesting chain effect of bubbles orientation is seen. The plasma is avalanched and formed to almost line geometry with increasing energy from the source. This corresponds to temporal and spatial plasma evolution. [6] The energy in the spot is not distributed continuously that is also shown in bubble formation - from the small to the bigger one.

The parts of bubbles are joined together. The part of small bubbles perishes independently on the rest. The multiple bubbles develop also in reverse focusing setup, but more compact.

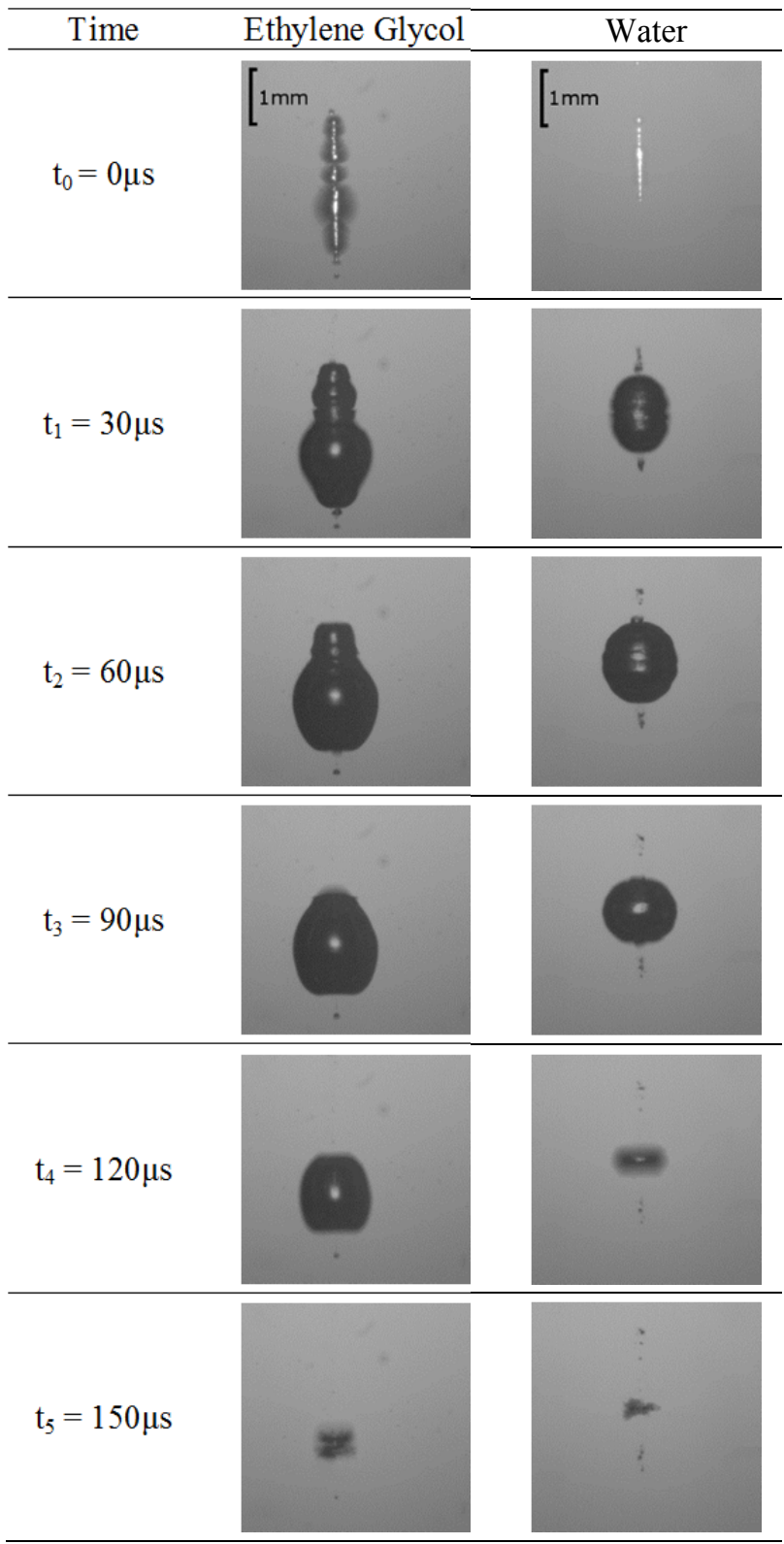

Fig. 6. Comparison of LIB generated bubble in Ethylene Glycol and water for laser input energy 5,6mJ.

They are further joined into big bubble that behaves as single one. As the bubbles were distributed at the beginning of cavitation process it is seen separated during the phase of implosion. Anyway the bubbles joined into single one, they are segregated again. In Figure 7, a primary show micro-jet creation through collapsing cavitation bubbles. It was well caught a specifically at time $t_{8}-t_{14}$. 

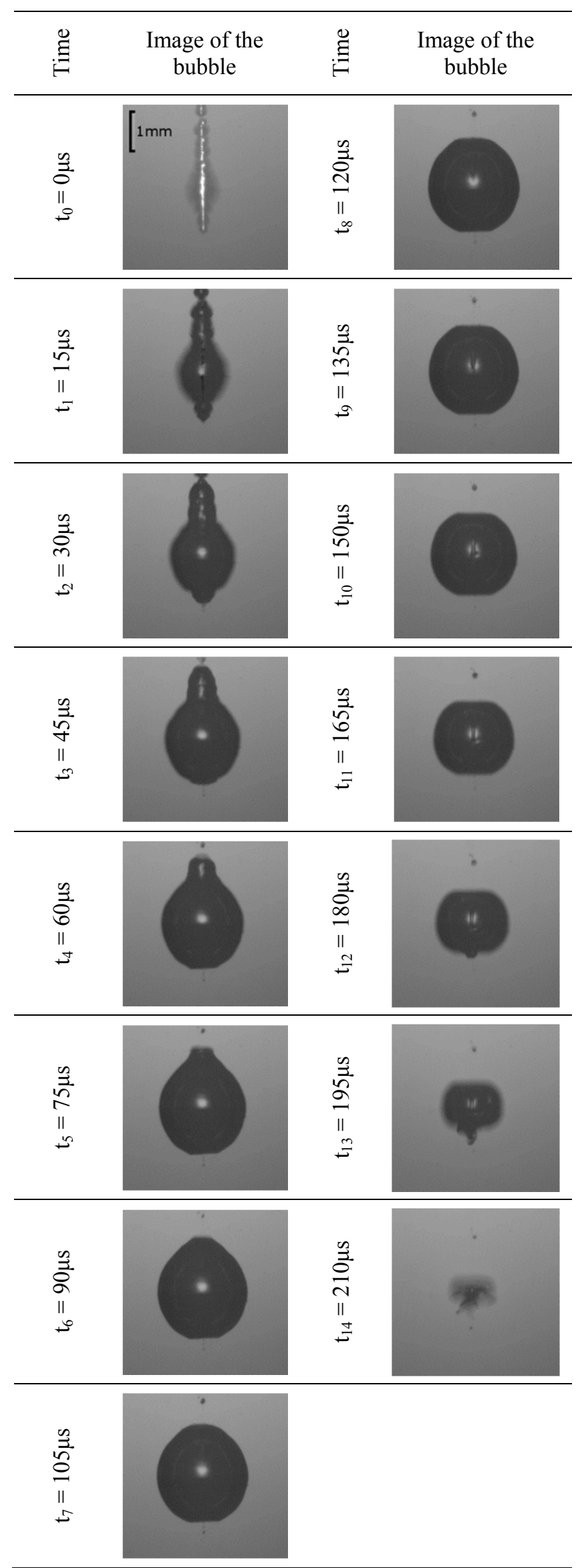

Fig. 7. LIB generated bubble in Ethylene Glycol for laser input energy $10,8 \mathrm{~mJ}$.

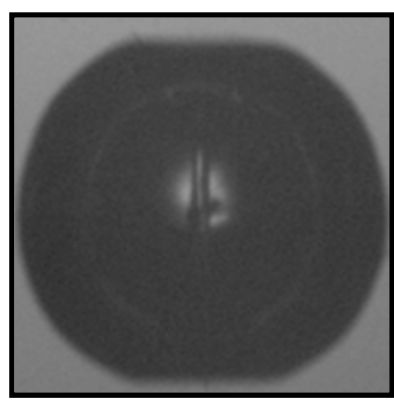

a) $t_{11}=165 \mu \mathrm{s}$

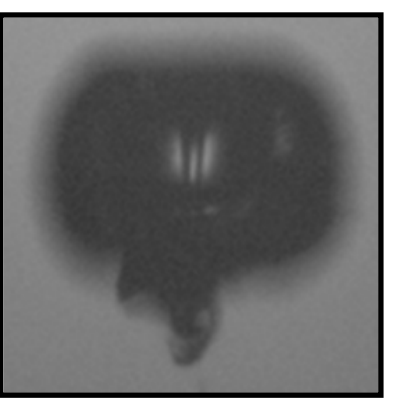

b) $t_{13}=195 \mu \mathrm{s}$
Fig. 8. Detail on micro-jet creation through collapsing cavitation bubble. LIB generated bubble in Ethylene Glycol for laser input energy $10,8 \mathrm{~mJ}$.

\section{Conclusions}

The visualization of cavitation bubbles is very important and useful tool in study of cavitation process. Here we use several type of liquid to study their advantages. We visualized the limits of the bubbles in their growth and size. This study enables us to get to know the importance of the suitable liquid selection and behaviour of bubble.

The volume in Figure 9 was obtained by calculating the pixel area of each captured image by the approximation of the sphere.

The curves in Figure 9 show the first phase of bubble life, with the exception of the Ethyl Alcohol curve. The Ethyl Alcohol Curve gets to zero at time of $90 \mu$ s and then begins to grow again as a result of the second phase of the bubble lifespan. This fully corresponds to the development of the bubble cavitation.

The plasma expansion is followed by the shock wave and during this sequence the liquid is vaporized and a cavitation bubble is growing. This bubble is filled with water vapour. The bubble collapse occurs during the second phase of the bubble lifespan. The collapse is caused by the reduction of interior pressure and cooling that is influenced by the external environment.

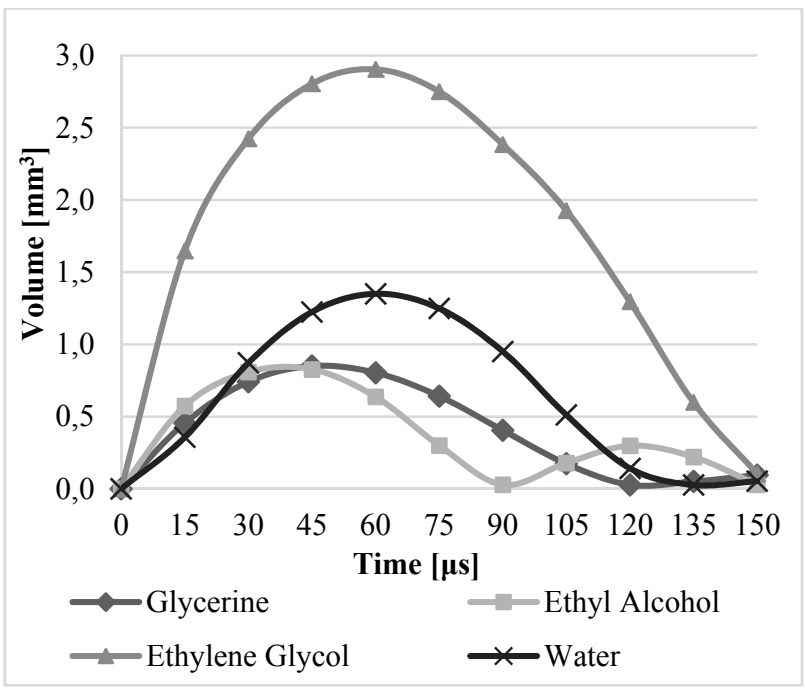

Fig. 9. Comparison of bubble size in different liquids. 
The bubble has been generated with the same energy of the laser $5,6 \mathrm{~mJ}$, where depending on the type of liquid was measured different gas volumes of bubbles. The largest bubble was formed in Ethylene Glycol as seen in Figure 9. It is further shown in Figure 9 that the bubble formed in Ethyl Alcohol had the collapse of the bubble most rapidly to $90 \mu \mathrm{s}$. The higher the viscosity of the liquid, the slower the bubble collapsed. Furthermore, in a liquid with higher absorbance was formed a bubble with a smaller volume of gas.

Here we managed to acquire a technique and procedure for evaluating bubble behavior over time that can be quantified and compared. This technique and procedure is based on image analysis. This first step is important for further investigation, but requires further optimization, such as approximating the bubble in a suitable shape.

\section{Acknowledgment}

The results of this project LO1201 were obtained through the financial support of the Ministry of Education, Youth and Sports in the framework of the targeted support of the "National Programme for Sustainability I", and the SGS project no. 21176/115. The authors gratefully thank to the support of Technology Agency of the Czech Republic (TA ČR) TH01020982 - Efficiency improvement of energy storage and ensuring grid stability by extending the operating range of pumped storage. Authors also acknowledge the institutional support of the Faculty of Mechatronics, Informatics and Interdisciplinary Studies of the Technical University of Liberec.

\section{References}

1. D. Jasikova, P. Schovanec, M. Kotek, M. Muller, V. Kopecky, Comparison of ultrasound and LIB generated cavitation bubble, EPJ Web of Conferences, 143, 2017.

2. D. Jasikova, P. Schovanec, M. Kotek, M. Muller, V. Kopecky, Experimental setup for laser-induced breakdown in aqueous media, Proceedings of SPIE, 10151, 2016.

3. D. Jasikova, M. Kotek, M. Muller, V. Kopecky, Single cavitation bubble interaction close to hydrophobic surface, International Journal of Mechanics, 11, 2017, 73-81.

4. D. Jasikova, M. Muller, M. Kotek, V. Kopecky, The synchronized force impact measurement and visualization of single cavitation bubble generated with LIB, International Journal of Mechanics, 9, 2015, 138-144.

5. Ch. E. Brennen, Cavitation and bubble dynamics, (Cambridge University Press, 2015)

6. P. K. Kennedy et.al, Prog.QuantElectr. 21, 3, (1997)

7. E. A. Brujan, et.al, J.Fluid Mech. 433, (2001)

8. W. Lauterborn, et.al, Archives of Acoustics 33, (2008)

9. A. Shima, Y. Tomita, Ingenieur-Archiv 51, (1981)

10. M. Gumulya, et. al, Bubbles in viscous liquids: Time dependent behaviour and wake characteristics, In Chemical Engineering Science, 144, 2016.

11. Chong Zhang, et. al, Dynamics of bubble formation in highly viscous liquids in a flow-focusing device, In Chemical Engineering Science, 172, 2017.

12. M. Kubala: Optical spectroscopy 1: Absorption spectroscopy. Olomouc 2011.

13. P. Šmejkal, I. Trejbalová, E. Urválková: Experiments of general and physical chemistry practicum. Prague 2007.

14. A. Vogel, S. Busch, U. Parlitz, J. Acoust. Soc. Am. 100, (1996)

15. A. Vogel, et. al, Applied Physics B: Lasers and Optics 68, (1999)

16. P. K. Kennedy et al., Prog. Quant. Electr. 21, 3, (1997) 